\title{
Effect of Different Methods for Rice Bran Oil Extraction on Crude Oil \%, Oil Quality and Bioactive Components of Some Rice Varieties
}

\author{
Khaled M.H. Abd El Salam ${ }^{1}$ and Dalia M.M. Tabl ${ }^{1}$
}

\begin{abstract}
Rice grains of ten rice varieties namely, Giza177, Giza 178, Sakha 101, Sakha 102, Sakha 104, Sakha 106, Giza 182, Yasmine, Sk2034(H1), and SK2046 (H2) were provided by Rice Research Program, Field Crop Research Institute, Agriculture Research Center, Sakha, Kafr ElSheikh, Egypt during 2018 and 2019 growing seasons. The experiment was laid out as split - plot design in a randomized complete block design (RCBD) with three replicates. The main - plot was devoted to tested ten rice varieties while sub - plot devoted to three different methods for rice bran oil extraction (hexane, isopropanol and screw press). The results were recorded on (hexane, isopropanol and screw press) as recorded by SES 2008 , were revealed that, there are significant difference among rice cultivars for all the studied characters, except peroxide value and free fatty acids\%. Comparison between means showed that, Giza 178 variety gave the highest values for crude oil\%, total phenolic content, tocotrienols, tocopherols and gama oryzanol in both studied seasons. Furthermore, Yasmin rice cultivar showed the highest values for saponification number in both seasons. The highest iodine value was recorded with Sakha 104 and SK 2034 H1 varieties in 2018 and2019 seasons, respectively. Extraction of rice bran oil by isopropanol showed the highest values for crude oil\%, iodine value, total phenolic content, tocotrienols, tocopherols and gama oryzanol in 2018 and 2019 seasons. Interaction between rice cultivars and different methods for extraction of rice bran oil revealed that using Giza 178 variety and extraction of rice bran oil by isopropanol solvent gives the highest values of crude oil \%, total phenolic content, tocotrienols ,tocopherols and gama oryzanol in 2018 and 2019 seasons. Correlation coefficient was highly significant and positive between crude oil and tocotrienols highly significant and positive correlation were found among total phenolic and each of tocotrienols, tocopherols and gama oryzanol, indicated that selection for total phenolic components enhancement each of tocotrienols, tocopherols and gama oryzanol. Finally, using Giza 178 as a donor can improve bran oil content in hybrid rice program.
\end{abstract}

Keywords: Rice, bran, bran oil, extraction methods, cultivars, Tocotrienols, Tocopherols and total phenolic compounds.

\section{INTRODUCTION}

In Egypt, the cultivated area of rice is about 1.3 million feddan which annually produces 5.27 million tons paddy rice producing 0.5 million tons of rice bran that could produce approximately 0.07 million tons of crude oil every year (RRTC, 2020). Rice bran is the main by product from rice processing industry. During the whiting process, which applies friction to the grain surface, the bran is removed from the kernel and usually accounts for about $10 \%$ of raw rice weight (Bhatnagar et al. 2014). Rice bran oil (also known as rice bran extract) is extracted from the germ and inner husk of rice, commonly called rice bran. The rice bran contains 10-23\% oil and (unlike oat bran) negligible amounts of water-soluble glucans and larger amounts of insoluble dietary fiber. It is notable for its high smoke point of $232{ }^{\circ} \mathrm{C}\left(450{ }^{\circ} \mathrm{F}\right)$ and its mild flavor, making it suitable for high temperature cooking methods such as stir frying and deep frying. It is popular cooking oil in several Asian countries, including Japan and Chine Many people have replaced their usual cooking oils with rice bran oil due to its higher nutritional value. It is the by-product of rice processing, accounted for $5-8 \%$ in grain weight. Rice bran contains grease (12-24\%), protein $(12-15 \%)$, cellulose $(9.8-10 \%)$, vitamins (VB1, VB2, VD) and minerals. Rice bran oil, contains the unsaturated fatty acids, accounted for $38-42 \%$ oleic acid and $32-35 \%$ linoleic acid. The linoleic acid is widely recognized as an essential fatty acid and decreased the blood cholesterol, prevents atherosclerosis and other health effects, so the rice bran oil is a kind of ideal edible vegetable oil (Wang, 2006; Marlene et al., 2005). High efficiency and maximize extracted oil components from rice bran become research hotspots in oil industry (Ghosh, 2007 and Xing et al., 2011). Rice bran oil is recognized as a type of healthy oil due to its content of palmitic (13.9-22.1\%), oleic (35.96-49.2\%), and linoleic acids (27.3-41.0\%) This oil also contains 95.6 $\%$ saponifiable lipids, such as glycolipids and phospholipids, and $4.2 \%$ unsaponifiable lipids, such as tocopherols, tocotrienols, $\gamma$-oryzanols, sterols and carotene oids. The tocopherol and tocotrienol content can be as high as $0.10-0.14 \%$, whereas the oryzanol

DOI: $10.21608 / A S E J A I Q J S A E .2020 .138118$

${ }^{1}$ Rice Technology Training Center (RTTC),Field Crops Research Institute,

Agricultural Research Center, Alexandria, Egypt.

Received November 25, 2020, Accepted, December 30, 2020. 
content ranges from approximately $0.9-2.9 \%$. Rice bran oil (RBO) is one of the best sources of tocols and oryzanol. Tocols (tocopherols and tocotrienols), a family of vitamin E-active substances, are wildly used plant-based ingredients in the food, cosmetics and pharmaceutical industries (Bramley et al., 2000; Abidi 2003). Studies suggested that tocotrienols are more efficient antioxidant, anti-cancer agent and inhibitor of cholesterol synthesis than the tocopherols (Singh et al., 2013). Gamma-oryzanol is one of the major components of RBO, and it is a mixture of several ferulate esters of triterpene alcohols and plant sterols (Friedman, 2013). Oryzanol is a well known antioxidant compound and is linked with decreasing serum and plasma cholesterol, decreasing platelet aggregation, and cholesterol absorption. Moreover oryzanol has been used in the treatment of hyperlipidemia, and disorders of menopause (Patel \& Naik 2004). Rice bran oil is unique among edible oils due to its rich source of nutritionally important phytoceuticals such as oryzanol, tocotrienols, tocopherols, etc. $\gamma$ - Oryzanol is one of the components of rice bran oil which have the potential to be used in pharmaceuticals, cosmoceutical and nutraceutical. It is present in rice bran oil at a level of 1-2\% where it serves as natural antioxidant. Rice bran oil is a rich source of natural antioxidants which can be used as free radical scavengers. It contains about $0.1-0.14 \%$ vitamin E components. The rice bran oil antioxidants are very efficient in reducing low density lipoprotein and total serum cholesterol. The present investigation aimed to determine effect of different methods of rice bran oil extraction on crude oil \%, its quality and bioactive components in some Egyptian rice cultivars.

\section{MATERIALS AND METHODS}

Rice grains of ten rice varieties namely, Giza177, Giza 178, Sakha 101, Sakha 102, Sakha 104, Sakha, 106 Giza 182 ,Yasmine, Sk2034(H1), and SK2046 (H2) were provided by Rice Research Program, Field Crops Research Institute, Agriculture Research Center, Sakha, Kafr El-Sheikh, Egypt during 2018 and 2019 growing seasons. The experiment was laid out as split - plot design in a randomized complete block design (RCBD) with three replicates. The main - plot was devoted to tested ten rice varieties while, sub - plot devoted to three different methods for rice bran oil extraction (hexane, isopropanol and screw press). Bran percentages of each cultivar were: $9.31,9.65,8.77,9.51$, $9.11,9.46,9.40,8.75,9.18$ and 9.05 , respectively. The bran was stabilized by heating rice bran in microwave at $850 \mathrm{~W}$ for 3 minutes according to the optimum methods explained by (Thanonkaew et al., 2012).

\section{Analytical methods:}

1- Oil extraction from rice bran samples:

1.1 Solvent extraction: rice bran oil was extracted by cold extraction technique using hexane and isopropanol according to (Chen and Bergman 2005).

1.2 Screw press extraction: rice bran oil was extracted by screw press according to (Amarasinghe and Gangodavilage 2004).

2- Percentage of free fatty acids (as oleic acid), iodine, saponification number and peroxide values were determined according to AOAC ( 1990).

3- Total phenolic content: Total phenolics of rice bran oil were extracted by methanol according to the method described by Steel et al. (2005) and determined colorimetrically using Folin-Ciocalteu's reagent method as described by Singleton et al. (1999).

4- Determination of vitamin E: Rice bran (1g) was extracted. Prior to HPLC analysis, the extracts were filtered through a $0.45 \mathrm{~mm}$ syringe filter. Tocopherol and tocotrienol were determined, using the reversed phase high performance liquid chromatography (RPHPLC), according to the method reported by Chen and Bergman (2005) with some modifications. The Shimadzu HPLC system (model L-6200A), equipped with a Photo diode array detector (Shimadzu, Japan) and a computer system, was used. Detection was operated at $292 \mathrm{~nm}$. The spectra from 250 to $600 \mathrm{~nm}$ were recorded for all peaks. The extracted samples were injected through a guardcolumn and separated on a C18 column $(4.60 \mathrm{x}$ $150 \mathrm{~mm}, 4 \mu \mathrm{m}$ ) (Phenomenex, USA). Gradient elution was then applied. Mobile phases A, B, and C were methanol, water and ethanol, respectively. The gradient was as follows: 0-12 $\min 92 \% \mathrm{~A}, 4 \% \mathrm{~B}$ and $4 \% \mathrm{C}: 12-25$ min linear gradient, from $4 \%$ B to $3 \%$ $\mathrm{B}$ and $4 \% \mathrm{C}$ to $5 \% \mathrm{C}$, with flow rate of $1.5 \mathrm{~mL} / \mathrm{min}$ and injection volume of $20 \mu \mathrm{L}$. The tocopherol and tocotrienol were detected at $292 \mathrm{~nm}$. Chromatograms were recorded, and peak areas were used to calculate the content of tocopherol and tocotrienol, against the standard curve of standards.

Analysis of variance and simple correlation coefficient were carried out and computed according to Gomez and Gomez (1984) using SAS program, version 8. Means were compared using least significant differences (LSD) at 0.05 level of probability.

\section{RESULTS AND DISCUSSIONS}

\section{1- Effect of rice cultivars:}

Performance of the studied rice cultivars is presented in Tables 1 and 2. 
Table 1. Influence of stabilized rice bran oil extracted by different methods on crude oil \% and its quality of some Egyptians rice cultivars in 2018 and 2019 seasons.

\begin{tabular}{|c|c|c|c|c|c|c|c|c|c|c|}
\hline \multirow[t]{2}{*}{ Treatments } & \multicolumn{2}{|c|}{ Crude oil \% } & \multicolumn{2}{|c|}{ Iodine value } & \multicolumn{2}{|c|}{ Peroxide Value } & \multicolumn{2}{|c|}{ Free Fatty acids \% } & \multicolumn{2}{|c|}{ Saponification No. } \\
\hline & 2018 & 2019 & 2018 & 2019 & 2018 & 2019 & 2018 & 2019 & 2018 & 2019 \\
\hline \multicolumn{11}{|c|}{ Cultivars (C) } \\
\hline G. 177 & 14.61 & 14.46 & 88.64 & 89.27 & 6.31 & 6.31 & 2.81 & 2.76 & 185.73 & 184.00 \\
\hline G.178 & 15.22 & 15.07 & 89.87 & 89.78 & 6.34 & 6.33 & 2.81 & 2.79 & 185.43 & 183.60 \\
\hline S.101 & 13.95 & 13.82 & 90.51 & 90.68 & 6.35 & 6.33 & 2.81 & 2.80 & 186.27 & 185.52 \\
\hline S.102 & 15.06 & 14.91 & 90.26 & 90.57 & 6.36 & 6.33 & 2.78 & 2.8 & 183.82 & 184.42 \\
\hline S.104 & 14.28 & 14.11 & 90.87 & 90.62 & 6.33 & 6.30 & 2.80 & 2.79 & 182.89 & 183.10 \\
\hline S.106 & 14.84 & 14.66 & 90.54 & 90.57 & 6.34 & 6.30 & 2.8 & 2.79 & 183.08 & 184.44 \\
\hline G.182 & 14.74 & 14.53 & 89.33 & 89.27 & 6.34 & 6.30 & 2.82 & 2.82 & 184.37 & 184.22 \\
\hline Yasmine & 13.77 & 13.59 & 88.32 & 88.65 & 6.37 & 6.36 & 2.81 & 2.79 & 188.33 & 188.03 \\
\hline $\mathrm{H} 1$ & 14.46 & 14.26 & 90.12 & 90.86 & 6.34 & 6.32 & 2.79 & 2.81 & 186.97 & 184.83 \\
\hline $\mathrm{H} 2$ & 14.13 & 13.95 & 88.88 & 89.90 & 6.37 & 6.31 & 2.81 & 2.78 & 184.82 & 182.32 \\
\hline L.S.D & 0.05 & 0.03 & 0.11 & 0.02 & N.S & N.S & N.S & N.S & 0.13 & 0.14 \\
\hline \multicolumn{11}{|c|}{ Methods of rice bran oil extraction (M ) } \\
\hline Hexane & 18.88 & 18.69 & 89.65 & 89.98 & 6.39 & 6.35 & 2.85 & 2.84 & 203.74 & 203.64 \\
\hline Isopropanol & 19.55 & 19.39 & 89.84 & 90.10 & 6.32 & 6.30 & 2.83 & 2.82 & 186.94 & 185.64 \\
\hline Screw press & 5.10 & 4.93 & 89.72 & 89.97 & 6.33 & 6.31 & 2.74 & 2.72 & 183.53 & 182.63 \\
\hline L.S.D & 0.25 & 0.46 & 0.01 & 0.01 & N.S & N.S & N.S & N.S & 2.13 & 2.03 \\
\hline \multicolumn{11}{|c|}{ Interaction } \\
\hline $\mathrm{C} * \mathrm{M}$ & $* *$ & $* *$ & N.S & N.S & N.S & N.S & N.S & N.S & N.S & N.S \\
\hline
\end{tabular}

$* *$ highly significant difference at 0.01 probability.

Table 2. Influence of stabilized rice bran oil extracted by different methods on bioactive components for some Egyptians rice cultivars in 2018 and 2019 seasons.

\begin{tabular}{|c|c|c|c|c|c|c|c|c|}
\hline \multirow[t]{2}{*}{ Treatments } & \multicolumn{2}{|c|}{$\begin{array}{l}\text { Total phenolic content } \\
\text { (mg GAE / g oil) }\end{array}$} & \multicolumn{2}{|c|}{$\begin{array}{l}\text { Tocotrienols } \\
(\mathrm{mg} / \mathrm{g})\end{array}$} & \multicolumn{2}{|c|}{$\begin{array}{l}\text { Tocopherols } \\
\text { (mg / g) }\end{array}$} & \multicolumn{2}{|c|}{$\begin{array}{c}\text { Gama oryzanol } \\
(\mathrm{mg} / \mathrm{g})\end{array}$} \\
\hline & 2018 & 2019 & 2018 & 2019 & 2018 & 2019 & 2018 & 2019 \\
\hline \multicolumn{9}{|c|}{ Cultivars (C) } \\
\hline Giza177 & 20.43 & 20.19 & 528.13 & 526.32 & 326.01 & 321.88 & 20.64 & 20.4 \\
\hline Giza 178 & 20.78 & 20.58 & 531.34 & 532.31 & 329.96 & 331.32 & 21.31 & 21.42 \\
\hline Sakha 101 & 20.27 & 20.11 & 526.68 & 525.24 & 324.26 & 320.56 & 20.83 & 20.57 \\
\hline Sakha 102 & 20.19 & 19.93 & 525.98 & 523.48 & 320.79 & 316.68 & 20.58 & 20.30 \\
\hline Sakha 104 & 19.82 & 19.53 & 525.09 & 525.43 & 322.67 & 317.05 & 20.26 & 20.22 \\
\hline Sakha 106 & 19.73 & 19.46 & 525.93 & 523.97 & 318.21 & 316.81 & 20.18 & 20.21 \\
\hline Giza 182 & 19.45 & 19.23 & 525.19 & 527 & 313.99 & 315.86 & 20.53 & 20.26 \\
\hline Yasmine & 20.17 & 19.92 & 523.13 & 523 & 320.32 & 318.12 & 20.97 & 20.69 \\
\hline $\mathrm{H} 1$ & 19.74 & 19.51 & 524.23 & 522.85 & 321.98 & 318.02 & 20.35 & 20.09 \\
\hline $\mathrm{H} 2$ & 19.34 & 19.1 & 524.08 & 525.35 & 316.15 & 311.01 & 20.11 & 20.03 \\
\hline L.S.D & 0.033 & 0.014 & 0.051 & 0.021 & 1.434 & 1.135 & 0.013 & 0.011 \\
\hline \multicolumn{9}{|c|}{ Methods of rice bran oil extraction (M) } \\
\hline Hexane & 18 & 17.8 & 489.03 & 488.57 & 293.15 & 289.48 & 14.86 & 14.72 \\
\hline Isopropanol & 22 & 21.75 & 590.68 & 590.47 & 362.83 & 361.71 & 27.87 & 27.72 \\
\hline Screw prees & 19.97 & 19.72 & 498.22 & 497.44 & 308.32 & 305 & 19 & 18.82 \\
\hline L.S.D & 1.27 & 1.05 & 6.12 & 5.76 & 5.73 & 6.31 & 2.45 & 2.11 \\
\hline \multicolumn{9}{|c|}{ Interaction } \\
\hline $\mathrm{C} * \mathrm{M}$ & $* *$ & $* *$ & $* *$ & $* *$ & $* *$ & $* *$ & $* *$ & $* *$ \\
\hline
\end{tabular}


Data revealed that there were significant differences between rice cultivars for all studied characteristics, except peroxide value and free fatty acids $\%$ in the two seasons. Comparison among means performance showed that variety Giza 178 recorded the highest values of crude oil\% (15.22 and $15.07 \%)$, Total phenolic content (20.78 and $20.58 \mathrm{mg} / \mathrm{GAE} / \mathrm{g}$ oil), Tocotrienols (531.34 and $532.31 \mathrm{mg} / \mathrm{g}$ ), Tocopherols (329.96 and $331.32 \mathrm{mg} / \mathrm{g}$ ) and gama oryzanol (21.31 and $21.42 \mathrm{mg} / \mathrm{g}$ ) during the two seasons, respectively.Furthermore, Yasmin rice cultivar showed the highest values of saponification no. (188.33 and 188.03) in the first and second seasons, respectively. Moreover, the highest values of iodine (90.87 and 90.86) were recorded with Sakha 104 variety and SK 2034 H1 (90.86) in 2018 and 2019 seasons, respectively. Variation between rice cultivars might be due to their genetic background (Abd El Bary 2012 and Abd El Salam 2017).

\section{2-Effect of different methods for rice bran oil extraction:}

Data in Tables $(1,2)$ showed that the effect of different methods of extraction for rice bran oil extraction showed significant differences for most studied characteristics, except peroxide value and free fatty acids \% in both studied seasons. Extraction of rice bran oil by isopropanol showed the highest values of crude oil\% (19.55 and $19.39 \%$ ), iodine value (89.84 and 90.10), total phenolic content (22 and $21.75 \mathrm{mg}$ GAE/g oil), tocotrienols (590.68 and $590.47 \mathrm{mg} / \mathrm{g}$ ), tocopherols (362.83 and $361.71 \mathrm{~m} / \mathrm{g}$ ) and gama oryzanol (27.87 and $27.72 \mathrm{mg} / \mathrm{g}$ ) in 2018 and 2019 seasons, respectively. However, the highest values of saponification number (203.74 and 203.64) were noticed with extraction of rice bran oil by hexane in both seasons, respectively. Peroxide value and free fatty acids didn't significantly affected with rice varieties in both seasons, due to extraction with hexane or isopropanol were done by cold extraction procedure which are similar to screw press procedure. These results were in agreement with Marei (2016). Furthermore, Extraction by isopropanol showed highest values for most studied characters due to increase in polarity. These results were in harmony with those obtained by Marei (2016).

\section{3- Interaction between rice cultivars and different methods for extraction of rice bran oil:}

Data in Tables (3 and 4) showed significant differences for interaction between rice cultivars and different methods for extraction of rice bran oil in both studied seasons.

Data revealed that using Giza 178 rice cultivar with extraction of rice bran oil by isopropanol solvent recorded highest values for crude oil \% (20.25 and $20.12 \%$ ), total phenolic content (22.87 and $22.66 \mathrm{mg}$ GAE/ g oil), tocotrienols (595.94 and $596.38 \mathrm{mg} / \mathrm{g}$ ), tocopherols (375.38 and $380.12 \mathrm{mg} / \mathrm{g}$ ) and gama oryzanol (28.93 and $28.76 \mathrm{mg} / \mathrm{g}$ ) in 2018 and 2019 seasons, respectively. On the other side, Sakha 106 variety combined with hexane method recorded the highest value for gama oryzanol (mg/g), while, the Giza 182 with hexane method recorded the lowest value for total phenolic content (mg GAE/g oil). Egyptian Yasmin with using Screw press method recorded the lowest value for crude oil \%, Egyptian hybrid Sakha 2034H1 with using hexane method recorded the highest value for tocotrienols. Finally, Egyptian Hybrid H2 combined hexane method recorded the lowest value for tocopherlos. From these results, it could be concluded that, to get highly quantity and quality of oil extraction of rice bran to different rice varieties, Isopropanol method should be used Zhu, et al (2010) and Rather, et al (2016).

\section{Correlation coefficient}

The data presented in Table (5) showed highly significant correlation among tocotrienols and each of crude oil\% (0.47) and total phenolic content (0.882), while, tocopherols had highly significant and positive correlated with each of total phenolic content (0.943) and tocotrienols (0.981). Moreover, the highly significant and positive relationship was found between gama oryzanol $(\mathrm{mg} / \mathrm{g})$ and each of total phenolic content (0.958), tocotrienols (0.971) and tocopherols (0.985). These results indicated that selection for total phenolic content will enhancement the tocotrienols, tocopherols and gama oryzanol through the rice breeding program. Similar results were obtained by Verma et al., (2015). 
Table 3. Crude oil\%, total phenolic content (mg GAE / g oil) and tocotrienols $(\mathrm{mg} / \mathrm{g})$ as affected by the interaction between rice cultivars and different extraction methods of rice bran oil in 2018 and 2019 seasons and combined data.

\begin{tabular}{|c|c|c|c|c|c|c|c|c|c|c|}
\hline \multirow{2}{*}{ Cultivars } & \multirow{2}{*}{$\begin{array}{l}\text { Methods of } \\
\text { rice bran oil } \\
\text { extraction }\end{array}$} & \multicolumn{3}{|c|}{$\begin{array}{c}\text { Crude oil } \\
(\%)\end{array}$} & \multicolumn{3}{|c|}{$\begin{array}{l}\text { Total phenolic content } \\
\text { (mg GAE / g oil) }\end{array}$} & \multicolumn{3}{|c|}{$\begin{array}{l}\text { Tocotrienols } \\
\quad(\mathrm{mg} / \mathrm{g})\end{array}$} \\
\hline & & 2018 & 2019 & combined & 2018 & 2019 & combined & 2018 & 2019 & combined \\
\hline \multirow{3}{*}{ Giza 177} & Hexane & 19.03 & 18.89 & 18.96 & 18.37 & 18.20 & 18.29 & 490.82 & 488.38 & 489.60 \\
\hline & Isopropanol & 19.60 & 19.45 & 19.53 & 22.58 & 22.25 & 22.42 & 592.36 & 590.20 & 591.28 \\
\hline & Screw press & 5.20 & 5.05 & 5.13 & 20.33 & 20.12 & 20.23 & 501.22 & 500.37 & 500.80 \\
\hline \multirow{3}{*}{ Giza 178} & Hexane & 19.57 & 19.41 & 19.49 & 18.75 & 18.58 & 18.67 & 494.81 & 495.72 & 495.27 \\
\hline & Isopropanol & 20.25 & 20.12 & 20.19 & 22.87 & 22.66 & 22.77 & 595.94 & 596.38 & 596.16 \\
\hline & Screw press & 5.85 & 5.69 & 5.77 & 20.72 & 20.51 & 20.62 & 503.26 & 504.82 & 504.04 \\
\hline \multirow{3}{*}{ Sakha 101} & Hexane & 18.25 & 18.16 & 18.21 & 18.26 & 18.11 & 18.19 & 489.31 & 488.39 & 488.85 \\
\hline & Isopropanol & 19.03 & 18.88 & 18.96 & 22.30 & 22.15 & 22.23 & 590.42 & 588.60 & 589.51 \\
\hline & Screw press & 4.56 & 4.41 & 4.49 & 20.25 & 20.06 & 20.16 & 500.31 & 498.72 & 499.52 \\
\hline \multirow{3}{*}{ Sakha 102} & Hexane & 19.41 & 19.28 & 19.35 & 18.14 & 17.91 & 18.03 & 488.32 & 485.20 & 486.76 \\
\hline & Isopropanol & 20.12 & 19.96 & 20.04 & 22.35 & 22.12 & 22.24 & 591.35 & 588.11 & 589.73 \\
\hline & Screw press & 5.66 & 5.49 & 5.58 & 20.07 & 19.76 & 19.92 & 498.26 & 497.14 & 497.70 \\
\hline \multirow{3}{*}{ Sakha 104} & Hexane & 18.62 & 18.50 & 18.56 & 17.88 & 17.50 & 17.69 & 489.57 & 487.26 & 488.42 \\
\hline & Isopropanol & 19.35 & 19.21 & 19.28 & 21.93 & 21.66 & 21.80 & 588.20 & 590.37 & 589.29 \\
\hline & Screw press & 4.87 & 4.62 & 4.75 & 19.65 & 19.42 & 19.54 & 497.51 & 498.66 & 498.09 \\
\hline \multirow{3}{*}{ Sakha 106} & Hexane & 19.28 & 19.11 & 19.20 & 17.60 & 17.40 & 17.50 & 490.31 & 488.45 & 489.38 \\
\hline & Isopropanol & 19.83 & 19.65 & 19.74 & 21.76 & 21.43 & 21.60 & 589.11 & 587.27 & 588.19 \\
\hline & Screw press & 5.42 & 5.21 & 5.32 & 19.82 & 19.56 & 19.69 & 498.37 & 496.20 & 497.29 \\
\hline \multirow{3}{*}{ Giza 182} & Hexane & 19.14 & 18.83 & 18.99 & 17.42 & 17.28 & 17.35 & 488.20 & 490.15 & 489.18 \\
\hline & Isopropanol & 19.75 & 19.60 & 19.68 & 21.35 & 21.12 & 21.24 & 590.66 & 592.36 & 591.51 \\
\hline & Screw press & 5.33 & 5.17 & 5.25 & 19.57 & 19.29 & 19.43 & 496.70 & 498.49 & 497.60 \\
\hline \multirow{3}{*}{ Yasmine } & Hexane & 18.11 & 17.90 & 18.01 & 18.26 & 18.13 & 18.20 & 487.11 & 490.56 & 488.84 \\
\hline & Isopropanol & 18.85 & 18.67 & 18.76 & 22.12 & 21.87 & 22.00 & 591.72 & 590.13 & 590.93 \\
\hline & Screw press & 4.36 & 4.20 & 4.28 & 20.14 & 19.76 & 19.95 & 490.55 & 488.32 & 489.44 \\
\hline \multirow{3}{*}{ Hybrid 1} & Hexane & 18.86 & 18.55 & 18.71 & 17.86 & 17.59 & 17.73 & 486.50 & 483.27 & 484.89 \\
\hline & Isopropanol & 19.47 & 19.31 & 19.39 & 21.50 & 21.33 & 21.42 & 588.36 & 590.10 & 589.23 \\
\hline & Screw press & 5.06 & 4.91 & 4.99 & 19.85 & 19.62 & 19.74 & 497.82 & 495.17 & 496.50 \\
\hline \multirow{3}{*}{ Hybrid 2} & Hexane & 18.48 & 18.3 & 18.39 & 17.44 & 17.30 & 17.37 & 485.32 & 488.36 & 486.84 \\
\hline & Isopropanol & 19.21 & 19.02 & 19.12 & 21.25 & 20.90 & 21.08 & 588.72 & 591.20 & 589.96 \\
\hline & Screw press & 4.70 & 4.52 & 4.61 & 19.34 & 19.10 & 19.22 & 498.20 & 496.48 & 497.34 \\
\hline L.S.D & & 0.01 & 0.02 & 0.01 & 0.02 & 0.01 & 0.02 & 1.31 & 1.88 & 1.59 \\
\hline
\end{tabular}


Table 4. Tocopherols ( $\mathrm{mg} / \mathrm{g})$ and gama oryzanol $(\mathrm{mg} / \mathrm{g})$ as affected by the interaction between rice cultivars and different extraction methods of rice bran oil in 2018 and 2019 seasons and combined data.

\begin{tabular}{|c|c|c|c|c|c|c|c|}
\hline \multirow[b]{2}{*}{ Cultivars } & \multirow{2}{*}{$\begin{array}{l}\text { Methods of rice } \\
\text { bran oil extraction }\end{array}$} & \multicolumn{3}{|c|}{ Tocopherols (mg / g) } & \multicolumn{3}{|c|}{ Gama oryzanol (mg / g) } \\
\hline & & 2018 & 2019 & combined & 2018 & 2019 & combined \\
\hline \multirow{4}{*}{ Giza 177} & Hexane & 295.37 & 293.21 & 294.29 & 14.81 & 14.50 & 14.66 \\
\hline & Isopropanol & 370.21 & 366.80 & 368.51 & 27.90 & 27.63 & 27.77 \\
\hline & Screw press & 312.45 & 305.62 & 309.04 & 19.22 & 19.07 & 19.15 \\
\hline & Hexane & 298.89 & 296.38 & 297.64 & 15.31 & 15.59 & 15.45 \\
\hline \multirow[t]{3}{*}{ Giza 178} & Isopropanol & 375.38 & 380.12 & 377.75 & 28.93 & 28.76 & 28.85 \\
\hline & Screw press & 315.62 & 317.45 & 316.54 & 19.68 & 19.92 & 19.80 \\
\hline & Hexane & 293.72 & 290.50 & 292.11 & 15.11 & 14.88 & 15.00 \\
\hline \multirow[t]{3}{*}{ Sakha 101} & Isopropanol & 371.86 & 368.35 & 370.11 & 28.13 & 27.82 & 27.98 \\
\hline & Screw press & 307.21 & 302.82 & 305.02 & 19.25 & 19.02 & 19.14 \\
\hline & Hexane & 290.65 & 287.30 & 288.98 & 14.81 & 14.60 & 14.71 \\
\hline \multirow[t]{3}{*}{ Sakha 102} & Isopropanol & 365.92 & 361.47 & 363.70 & 27.90 & 27.55 & 27.73 \\
\hline & Screw press & 305.81 & 301.28 & 303.55 & 19.03 & 18.76 & 18.90 \\
\hline & Hexane & 296.65 & 290.82 & 293.74 & 14.66 & 14.91 & 14.79 \\
\hline \multirow[t]{3}{*}{ Sakha 104} & Isopropanol & 360.82 & 356.13 & 358.48 & 27.50 & 27.33 & 27.42 \\
\hline & Screw press & 310.53 & 304.19 & 307.36 & 18.62 & 18.43 & 18.53 \\
\hline & Hexane & 293.70 & 290.33 & 292.02 & 14.33 & 14.56 & 14.45 \\
\hline \multirow{3}{*}{ Sakha 106} & Isopropanol & 356.12 & 359.20 & 357.66 & 27.31 & 27.57 & 27.44 \\
\hline & Screw press & 304.80 & 300.89 & 302.85 & 18.90 & 18.51 & 18.71 \\
\hline & Hexane & 288.54 & 285.60 & 287.07 & 15.05 & 14.46 & 14.76 \\
\hline \multirow[t]{3}{*}{ Giza 182} & Isopropanol & 350.85 & 354.75 & 352.80 & 28.11 & 27.74 & 27.93 \\
\hline & Screw press & 302.57 & 307.23 & 304.90 & 18.42 & 18.57 & 18.50 \\
\hline & Hexane & 294.42 & 291.41 & 292.92 & 15.13 & 14.82 & 14.98 \\
\hline \multirow[t]{3}{*}{ Yasmine } & Isopropanol & 356.80 & 360.21 & 358.51 & 28.42 & 28.13 & 28.28 \\
\hline & Screw press & 309.75 & 302.75 & 306.25 & 19.36 & 19.11 & 19.24 \\
\hline & Hexane & 291.66 & 288.79 & 290.23 & 14.78 & 14.55 & 14.67 \\
\hline \multirow[t]{3}{*}{ Hybrid 1} & Isopropanol & 362.73 & 357.82 & 360.28 & 27.36 & 27.21 & 27.29 \\
\hline & Screw press & 311.55 & 307.46 & 309.51 & 18.92 & 18.50 & 18.71 \\
\hline & Hexane & 287.89 & 280.43 & 284.16 & 14.61 & 14.36 & 14.49 \\
\hline \multirow[t]{2}{*}{ Hybrid 2} & Isopropanol & 357.62 & 352.29 & 354.96 & 27.12 & 27.43 & 27.28 \\
\hline & Screw press & 302.93 & 300.31 & 301.62 & 18.60 & 18.31 & 18.46 \\
\hline L.S.D & & 1.16 & 1.56 & 1.36 & 0.04 & 0.02 & 0.03 \\
\hline
\end{tabular}

Table 5. The relationship among the studied characters of some rice genotypes from the combined data during two seasons.

\begin{tabular}{lcccc}
\hline Traits & $\begin{array}{c}\text { Crude oil } \\
\text { \% }\end{array}$ & $\begin{array}{c}\text { Total phenolic content } \\
\text { (mg GAE / g oil) }\end{array}$ & $\begin{array}{c}\text { Tocotrienols } \\
\text { (mg / g) }\end{array}$ & $\begin{array}{c}\text { Tocopherols } \\
\text { (mg / g) }\end{array}$ \\
\hline Total phenolic content (mg GAE / g oil) & 0.056 & & & \\
Tocotrienols (mg / g) & $0.466^{* *}$ & $0.882^{* *}$ & & \\
Tocopherols (mg / g) & 0.348 & $0.943^{* *}$ & $0.981^{* *}$ & \\
Gama oryzanol (mg / g) & 0.247 & $0.958^{* *}$ & $0.971^{* *}$ & $0.985^{* *}$ \\
\hline
\end{tabular}

\section{CONCLUSION}

The present study indicated that using Giza 178 rice cultivar with extraction of rice bran oil by isopropanol method gave the highest values for crude oil $\%$ and bioactive components which have various beneficial effects on human health.

\section{REFERENCES}

Abd El Bary, Doaa A.A. 2012. Qualitative and quantitative assessment for bran and bran oil of some Egyptian rice varieties, A Thesis of ph. D. Public Health Sciences (Food Analysis), High Institute of Public Health, Alex., Egypt.

Abd El Salam, K. M.H. 2017. Influence of stabilization methods on rice bran oil of some egyptian rice cultivars, Alex. Sci. exch.J., 62(2): 185-193. 
Abidi, S. L. 2003. Tocol-derived minor constituents in selected plant seed oils. Journal of the American Oil Chemists' Society. 80(4):327- 333.

Amarasinghe B.M.W.P.K. and N.C. Gangodavilage. 2004. Rice bran oil extraction in Sri Lanka: Data for process equipment design. Food Soup J., 82: 54-59.

AOAC 1990. Official Methods of Analysis, 11th Ed. Association of Official Analytical Chemists. Washington D.C, USA.

Bhatnagar, AS., DS.Prabhakar, PKP Kumar., RGR. Rajan and AGG. Krishna. 2014. Processing of commercial rice bran for the production of fat nutraceutical rich rice brokens, rice germ and pure bran. J. Food Sci. Technol. 58:306311.

Bramley, P.M. I.Elmadfa, A. Kafatos, F.J. Kelly,Y. Manios, H.E. Roxborough, W.Schuch, P. J. A. Sheehy, \& K. H. Wagner .2000.Vitamin E. Journal of the Science of Food andAgriculture,80(7),913938.http://dx.doi.org/10.1002/(SI CI) $10970010(20000515) 80: 7<913:$ :AIDJSFA600>3.0.CO;2-3.

Chen, N. and C.J. Bergman. 2005. A rapid procedure for analysing rice bran tocopherol, tocotrienol and $\gamma$-oryzanol contents. J. of Food Composition and Analysis, 18: 139151.

Friedman, M. 2013. Rice brans, rice bran oils, and rice hulls: composition, food and industrial uses, and bioactivities in humans, animals, and cells. Journal of Agricultural and Food Chemistry. 61(45): 10626-10641.

Ghosh, M. 2007. Review on recent trends in rice bran oil processing. J. Am. Oil Chem. Soc., 84: 315-324.

Gomez,K.A. and A.A. Gomez. 1984. Statistical Procedures for Agricultural Research. An International Rice Research Institute Book. John Willey and sons Inc., New York.

Marei, A.M.A. 2016. Some technological processes affecting rice bran stability for functional components recovery. A Thesis of Ph.D., Fac. of Agric. (Saba Basha), Alex. Univ., Egypt.

Marlene, M.M., T. Richard, M. Silvia and L. Michael . 2005. Rice bran oil, not fiber, lowers cholesterol in humans. Am. J. Clin. Nutr., 81: 64-68.
Patel, M., and Naik, S. N. 2004. Gamma-oryzanol from rice bran oil: a review. Journal of Scientific and Industrial Research. 63:569-578.

Rather, T. A., M.A. Malik and A. H. Dar. 2016.Physical, milling, cooking and pasting characteristics of different rice varieties grown in the valley of Kashmir India. Cogent Food\& Agric., 2:1.1178694.

RRTC. 2020. Technical recommendations to the rice crop. Rice Program-Research and Training Centre in rice- ARC, Egypt.

Singh, V. K., L. A Beattie and T. M. Seed. 2013. Vitamin E: tocopherols and tocotrienols as potential radiation countermeasures. Journal of Radiation Research, 54(6): 973-988.

Singleton, V.L., R. Orthofer and R.M. Lamuela-Raventos. 1999. Analysis of total phenols and other oxidation substrates and antioxidants by means of Folin-Ciocalteau reagent. Metho. Enzymol., 299: 152 - 178.

Steel, C.J., M.C. Dobarganes and D. Barrera-Arellano. 2005. The Influence of natural tocopherols during thermal oxidation of refined and partially hydrogenated soybean oils. Grasas Aceites. 56:46 - 52.

Thanonkaew, A., S. Wongyai, D. J. McClements and E. A. Decker. 2012. Effect of stabilization of rice bran by domestic heating on mechanical extraction yield, quality, and antioxidant properties of cold-pressed rice bran oil (Oryza saltiva L.), LWT - Food Science and Technology, 48: 231-236.

Wang, Y. B. 2006. The current research situation and development trend of functional component in rice bran oil. Food Nutr. China, 1: 17-20.

Xing, L., F.M. Zhao, Y.F. Cao, Y.X. Chen and D. Su. 2011.. Research progress on processing technology of rice bran oil. Food Nutr. China, 17: 35- 37.

Zhu, F., Y. Cai; J. Bao and H.Corke. 2010. Effect of Yirridation on phenolic compound in rice grain. Food Chem., 120:74-77. 


\section{الملخص العربي}

تأثير الطرق المختلفة لأستخراج زيت رجيعة الأرز على نسبة الزيت الخام، جودة الزيت والمكونات النشطة بيولوجيا في بعض أصناف الأرز

خالد مصطفى حمدي عبد السلام, داليا محمد محمد طبل

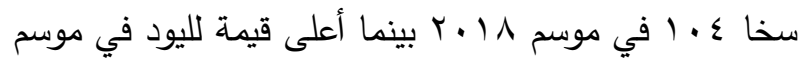

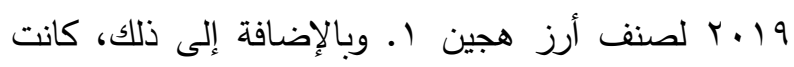

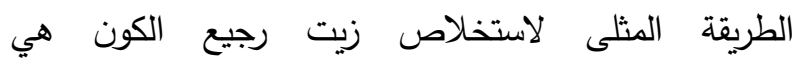

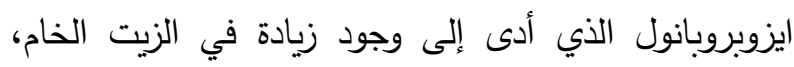

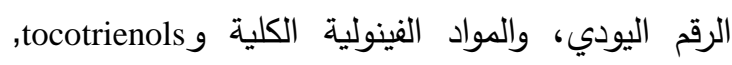
tocopherol

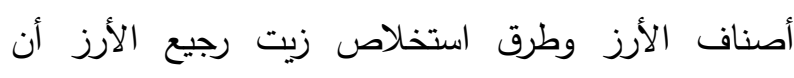

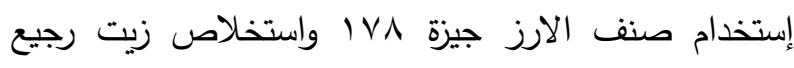
الأرز بايزوبروبانول أعطى أعلى القيم لنسبة الزيت الخام

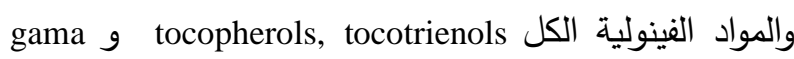

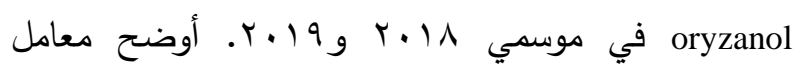
الارتباط وجود ارتباطا معنويا موجبا بين محتوى الزيت من ون الزيح

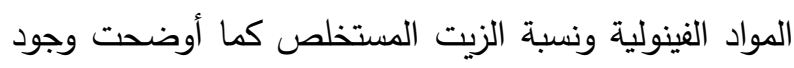

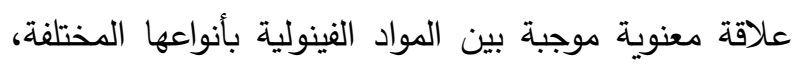

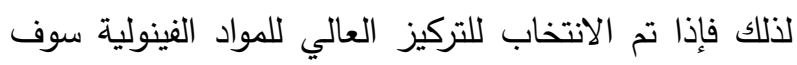
يعمل علي زيادة تركيز الفينولات المتعدد ونسبة الزيت.
تم الحصول على تقاوى معتمدة تم حصادها في موسمي

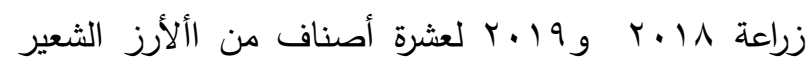

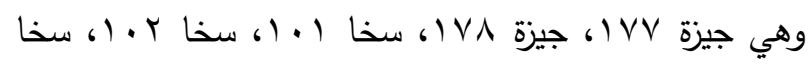

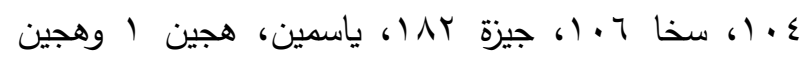

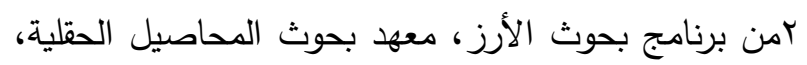

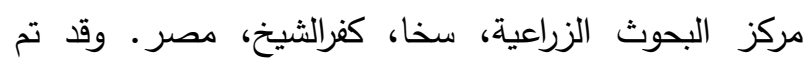
إستخدام تصميم القطع المنثقة مرة واحدة بثلاث مكررات حيث وزعت أصناف الأرز في القطع الرئيسية بينما كانت طرق استخلاص زيت رجيع الكون في القطع الفرعية (هكسان وايزوبروبانول والعصر الميكانيكي). وأوضحت النتائج وجود

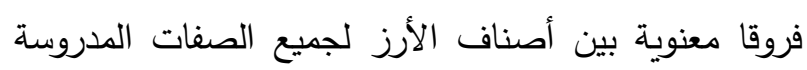

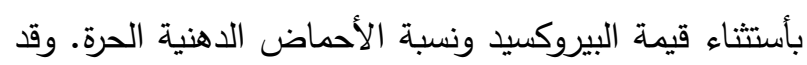
سجلت أعلى القيم لنسبة الزيت الخام، والمواد الفينولية الكلية gama oryzanolg tocotrienols, tocopherol الأرزجيزة VA في كلا موسمي الدراسة. علاوة على ذلك، أظهر صنف ياسمين أعلى القيم لصفة التصبن في كلي كلا الموسمين. بينما، تم تسجيل أعلى قيمة لليود مع صنف الأرز 